\title{
Transforming dark photons into sustainable energy
}

\author{
Md. Faruque Hossain ${ }^{1,2}$
}

Received: 2 August 2017 / Accepted: 14 December 2017 / Published online: 25 January 2018

(c) The Author(s) 2018. This article is an open access publication

\begin{abstract}
To produce energy from dark photons $\gamma^{-}$, colliding it with Higgs boson particle $\mathrm{BR}\left(\mathrm{H} \rightarrow \gamma \gamma^{-}\right)$under extreme relativistic conditions (ERCs) has been proposed. The Higgs boson $\mathrm{BR}\left(\mathrm{H} \rightarrow \gamma \gamma^{-}\right)$gets excited at extreme relativistic conditions and its quantum field has an extremely short-range weak force which can create electromagnetic field. Thus, it has been assumed that the induction of an electrically charged particle dark photon $\gamma^{-}$(non-energy level photon), into these extreme relativistic conditions will create an energy level photon, here named the Hossain dark photon (HDP). To confirm this HDP formation via a dark photon quantum interaction with a Higgs boson particle $\mathrm{BR}\left(\mathrm{H} \rightarrow \gamma \gamma^{-}\right)$, a series of mathematical modeling tests using MATLAB software has been performed. Interestingly, these mathematical models revealed that the presence of extra relativistic conditions does transform the dark photon $\gamma^{-}$into an HDP for $N_{\text {eff }}=4.08_{-0.68}^{+0.71}$ at a $95 \%$ confidence limit considering the parameters of $c_{\text {eff }}^{2}=0.312 \pm 0.026$ and $c_{\text {vis }}^{2}=0.29_{-0.16}^{+0.21}$, respectively. Thereafter, the feasible HDP photon dynamics is also modeled using the atomic spectra contour maps of the Hamiltonian $\left(H=\sum \omega_{c i} a_{i}^{\dagger} a_{i}+\sum_{K} \omega_{k} b_{k}^{\dagger} b_{k}+\sum_{i k}\left(V_{i k} a^{\dagger} b_{k}+V_{i k}^{*} b_{k}^{\dagger} a_{i}\right)\right)$ which suggested that formation of HDP by a Higgs boson $\left[\mathrm{BR}\left(\mathrm{H} \rightarrow \gamma \gamma^{-}\right]\right.$particle reaction under extreme relativistic conditions does form high energy level particle to produce electricity.
\end{abstract}

Keywords Dark photon $\cdot$ Higgs boson $\left[\mathrm{BR}\left(\mathrm{H} \rightarrow \gamma \gamma^{-}\right]\right.$particle $\cdot$ Ultra-relativistic conditions $\cdot$ Hossain dark photon (HDP) Renewable energy

\section{Introduction}

To analyze the ability of dark photons to create energy, I have presented a mass constraint model, along with key systematic parameters and Planck priors that constrain the masses to $\sum m \nu=0.041 \mathrm{eV}$ at the $1-\sigma$ level which is comparable to the Stage 4 CMB [1.8]. These constraints are derived by marginalizing the number of relativistic degrees of freedom $\left(N_{\text {eff }}\right)$, which are the degenerative function of the neutrino mass $[1,2]$. Thus, using the same function in MATLAB, I have explored the ability of the LSST-era test "standard" models for the HDR activation. Several studies interestingly revealed that approximately $80 \%$ of all mass in the solar system is made of dark matter (axions, neutralinos, photinos, and fifth force), comprises of weakly interacting

Md. Faruque Hossain

faruque55@aol.com

1 Department of Civil and Urban Engineering, New York University, 6 Metro Tech Center, Brooklyn, NY 11201, USA

2 Green Globe Technology, 4323 Colden Street 15 L, Flushing, New York 11355, USA massive particles (WIMPs) which has the possibility to act as the energy state level matter to create energy [3-5]. Thus, in this model, a set of mathematical evidence has been performed to determine the $N_{\text {eff }}$ measurements to detect the mass of HDP radiation at $1-\sigma$ level as $0.162 \mathrm{eV}$ for fermionic, and $0.137 \mathrm{eV}$ for dark radiation, expressing the following predictions at extreme relativistic condition, [6]:

$\rho_{\text {rad }}=\left[1+\frac{7}{8}\left(\frac{4}{11}\right)^{4 / 3} N_{\text {eff }}\right] T_{\gamma}$

where $\rho_{\gamma}$ represents dark matter energy density under extreme temperatures $T_{\gamma}$ and the effective number of relativistic degrees of freedom $N_{\text {eff }}$ of non-instantaneous neutrino decoupling from the primordial photon-baryon plasma (see, e.g., 1). Further, these theoretical predictions were clarified by the physical mechanisms of the Higgs boson, which confirm the HDP photon activation under extreme relativistic conditions by the following series of equations [7-9]:

$\dot{\delta}_{v}=\frac{\dot{a}}{a}\left(1-3 C_{\mathrm{eff}}^{2}\right)\left(\delta_{v}+3 \frac{\dot{a}}{a} \frac{q_{v}}{k}\right)-k\left(q_{v}+\frac{2}{3 k} \dot{h}\right)$, 
$\dot{q}_{v}=k C_{\mathrm{eff}}^{2}\left(\delta_{v}+3 \frac{\dot{a}}{a} \frac{q_{v}}{k}\right)-\frac{\dot{a}}{a} q_{v}-\frac{2}{3} k \pi_{v}$

$\dot{\pi}_{v}=3 c_{\text {vis }}^{2}\left(\frac{2}{5} q_{v}+\frac{8}{15} \sigma\right)-\frac{3}{5} k F_{v}, 3$,

$\frac{2 l+1}{k} \dot{F}_{v, l}-l F_{v, l-1}=-(l+1) F_{v, l+1}, l \geq 3$,

where $c_{\text {eff }}$ is the photon speed, and $c_{\text {vis }}$ represents the viscosity speed, which is equivalent to $c_{\text {eff }}^{2}=c_{\text {vis }}^{2}=1 / 3$.

Consequently, this mathematical analysis has been described as the evidence of the transformation of dark photons and converts it into electricity through a semiconductor under ultrarelative conditions. Just because of the electrons of dark photons have the negative charge and two electrons that can interact with each other through their electromagnetic forces create electron-positron pairs under extreme relativistic conditions by ion-ion collisions $[10,11]$. Therefore, the transformation of the proposed HDP will have its momentum to convert it into energy.

\section{Methods and materials}

\section{Dark photon modeling}

In this model, I initially compared the fifth-dimensional standard set of parameters of the densities of dark photons $\Omega_{b}$ and $\Omega_{c}$ considering the proportional rates with respect to the angular distance of the uncoupling $\theta$ at the optic intensity of re-ionization $\tau$. In addition, the scalar spectral index $n_{\mathrm{s}}$ and the average normalized spectrum $A_{\mathrm{S}}$ at $k=0.002 \mathrm{MPC}^{-1}$ were also calculated. Thus, the functional number for the relativistic degrees of freedom of photon $N_{\text {eff }}$, the functional speed $c_{\text {eff }}^{2}$ and the viscosity $c_{\text {vis }}^{2}$ have been identified to confirm the emissions of dark photon as $N_{V}^{S}=N_{\text {eff }}$, with respect to the perturbation parameters $c_{\text {vis }}$ and $c_{\text {eff }}$ for extreme relativistic conditions, which is done by calculating $c_{\text {eff }}^{2}=c_{\text {vis }}^{2}=1 / 3$ [12-14]. Considering the SZ amplitude $A_{\mathrm{SZ}}$, the amplitude of the clustered point sources $A_{\mathrm{C}}$ and the amplitude of the Poisson distributed point sources $A_{\mathrm{p}}$, I have also marginalized the contribution of the point sources of the ACT and SPT to the release of dark photon force energy by calculating the modification of Newton's kinetic energy equation using MATLAB software, as expressed below:

$$
\begin{aligned}
T_{k \cdot e} & =\int_{0}^{v} F \cdot \mathrm{d} s=\int_{0}^{v} \frac{\mathrm{d} p}{\mathrm{~d} t} \cdot \mathrm{d} s \\
& =\int_{0}^{v} \frac{\mathrm{d} s}{\mathrm{~d} t} \mathrm{~d} p=\int_{0}^{v} v \mathrm{~d} p=\int_{0}^{v} \mathrm{~d}(p v)-\int_{0}^{v} p \mathrm{~d} v .
\end{aligned}
$$

Since $\mathrm{d}(p v)=v \mathrm{~d} p+p \mathrm{~d} v$, by differentials

$\Rightarrow v \mathrm{~d} p=\mathrm{d}(p v)-p \mathrm{~d} v$

$\Rightarrow T_{k . e}=\int_{0}^{v} v \mathrm{~d} p=\int_{0}^{v} \mathrm{~d}(p v)-\int_{0}^{v} p \mathrm{~d} v$

$=\int_{0}^{v} v \mathrm{~d} p=\int_{0}^{v} \mathrm{~d}(p v)-\int_{0}^{v}(m v) \mathrm{d} v$, definition of momentum

$=p v-\int_{0}^{v}\left(\frac{m_{0} v}{\sqrt{1-\frac{v^{2}}{c^{2}}}}\right) \mathrm{d} v$, anti-derivative and relativistic momentum

$=(m v) v-m_{0} \int_{0}^{v}\left(\frac{v}{\sqrt{1-\frac{v^{2}}{c^{2}}}}\right) \mathrm{d} v, m_{0}$ rest mass is constant

$=m v^{2}-m_{0} \int_{0}^{v}\left(\frac{v}{\sqrt{1-\frac{v^{2}}{c^{2}}}}\right) \mathrm{d} v$

$=m v^{2}-m_{0} \cdot \frac{c}{c} \int_{0}^{v} \frac{v}{\sqrt{1-\frac{v^{2}}{c^{2}}}} \mathrm{~d} v$

$c=$ speed of light, a universal constant

$=m v^{2}-m_{0} c \int_{0}^{v} \frac{v}{\sqrt{1-\frac{v^{2}}{c^{2}}}} \mathrm{~d} v$, moving constant cinside the integral

$T_{k . e}=m v^{2}-m_{0} c \int_{0}^{v} \frac{v}{c^{2}-v^{2}} \mathrm{~d} v$, moving constant $c$ inside the radical

Further, a combination of the oscillations of the measurable factors for each component (clustered and Poisson distributed) of the dark photons modeling has also been identified by the SZ oscillation in the WMAP, SPT and ACT templates using $[15,16]$ MATLAB with an adaptation of the Newtonian work-energy equation. Here, the mass of $m_{0}$ at rest at the origin of the $x$-axis is used to transform a dark photon into a HDP to create energy, as described by the following equations: 


$$
\begin{aligned}
& \Delta K=W=\int_{r_{0}}^{r_{1}} F \cdot \mathrm{d} r \\
& =\int_{t_{0}}^{t_{1}} \frac{\mathrm{d}}{\mathrm{d} t}(\gamma m \mathbf{v}) \cdot \mathbf{v} \mathrm{d} t \\
& =\gamma m \mathbf{v}^{2} \mathbf{v} \mid \begin{array}{l}
t_{1} \\
t_{0}
\end{array}-\int_{t_{0}}^{t_{1}} \gamma m \mathbf{v} \cdot \frac{\mathrm{d} \mathbf{v}}{\mathrm{d} t} \mathrm{~d} t \\
& =\gamma m v^{2} \mid \begin{array}{l}
\boldsymbol{t}_{1} \\
\boldsymbol{t}_{0}
\end{array}-m \int_{v_{0}}^{v_{1}} \gamma v \mathrm{~d} v \\
& =m\left(\gamma v^{2} \mid \begin{array}{l}
t_{1} \\
t_{0}
\end{array}-c^{2} \int_{v_{0}}^{v_{1}} \frac{2 v / c^{2}}{2 \sqrt{1-v^{2} / c^{2}}} \mathrm{~d} v\right) \\
& =m\left(\frac{v^{2}}{\sqrt{1-v^{2} / c^{2}}}+c^{2} \sqrt{1-v^{2} / c^{2}}\right) \mid \begin{array}{l}
t_{1} \\
t_{0}
\end{array} \\
& =\frac{m c^{2}}{\sqrt{1-v^{2} / c^{2}}} \mid \begin{array}{l}
t_{1} \\
t_{0}
\end{array} \\
& =\gamma m c^{2} \mid \begin{array}{l}
t_{1} \\
t_{0}
\end{array}=\gamma_{1} m c^{2}-\gamma_{0} m c^{2} \\
& =\gamma_{1} m c^{2}-0\left(y_{0} \text { is equal to } 0 \text { since, at time } t_{0} \text {, no energy is produced }\right) \\
& =\gamma_{1} m c^{2}
\end{aligned}
$$
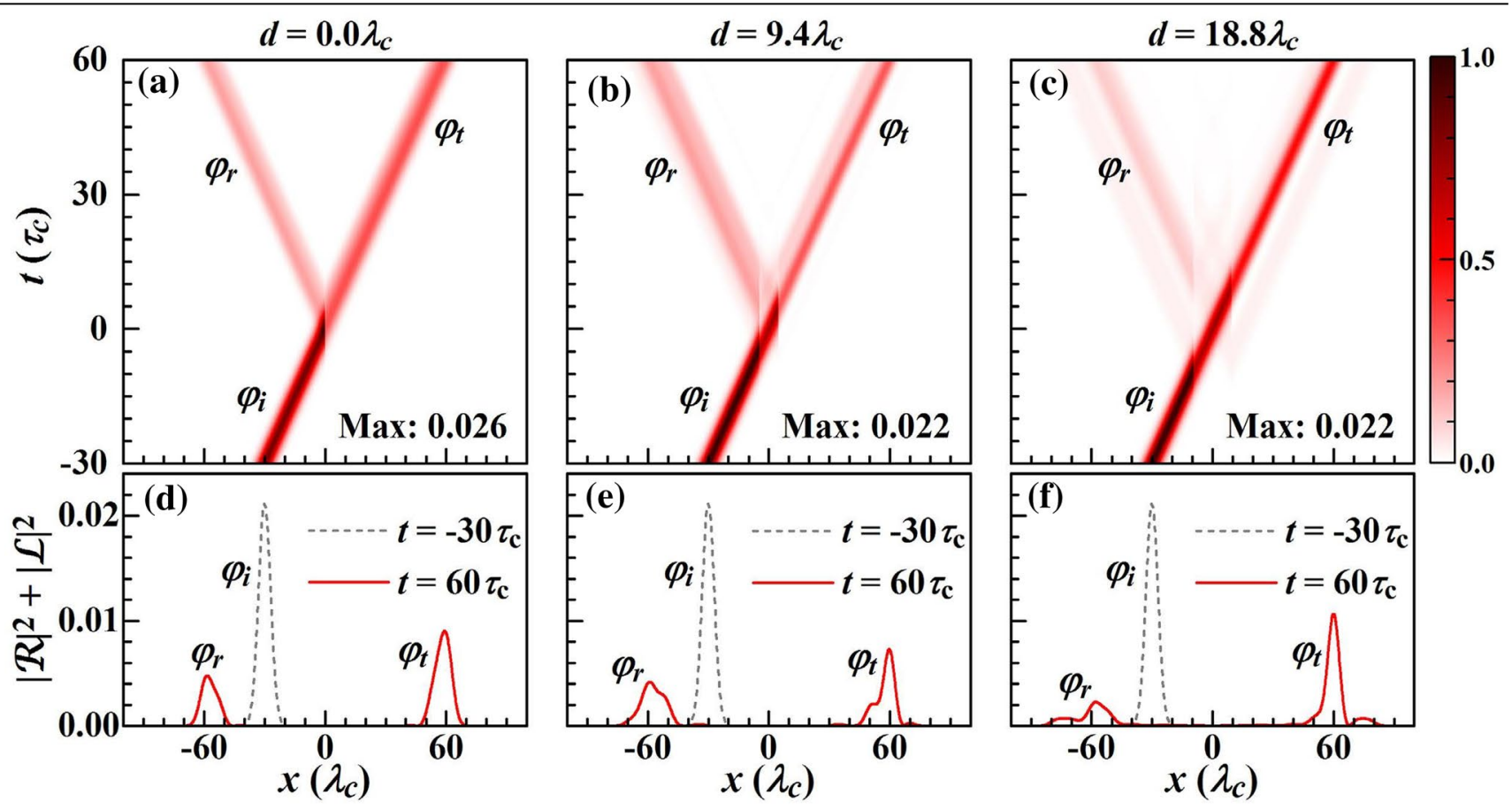

Fig. 1 a-c The contour maps of the dark photon probability densities, normalized by their maximum values are mapped as functions of $x$ and $t$. d-f The probability distributions of the incident $\left(\varphi_{\mathrm{i}}\right)$, reflected $\left(\varphi_{\mathrm{r}}\right)$, and transmitted $\left(\varphi_{\mathrm{t}}\right)$ pulses 


\section{Dark photon transformation}

To identify the HDP transformation, I have further proposed the dark photon dynamics at the nanoscale through point break waveguides embedded in a semiconductor circuit at extreme relativistic conditions. For this calculation, I have treated both the semiconductor and the point break waveguides as reservoirs of photons. Therefore, the nanoscale point breaks in the semiconductor panel satisfy the purely electron dynamics for continuous state photons with the adaptation of a Newtonian work-energy equation considering the atomic spectra and contour maps; thus, the Hamiltonian is expressed as $[5,6,17]$ :

$$
H=\sum \omega_{c i} a_{i}^{\dagger} a_{i}+\sum_{K} \omega_{k} b_{k}^{\dagger} b_{k}+\sum_{i k}\left(V_{i k} a^{\dagger} b_{k}+V_{i k}^{*} b_{k}^{\dagger} a_{i}\right)
$$

where $a_{i}\left(a_{i}^{\dagger}\right)$ represents the driver of the nanoscale point break mode, $b_{k}\left(b_{k}^{\dagger}\right)$ represents the driver of the photodynamic modes of the nanoscale structures of the dark photons, and the coefficient $V_{i k}$ represents the magnitude of the dark photonic mode among the nanoscale break points and dark photon nanoscale structures (Fig. 1).

Considering the initial dark photonic structures in an equilibrium state, I integrate the whole dark photonic reservoir structure noting the excited coherent state of the dark photons in the semiconductor. This is expressed by the following master equation [18-20]:

$$
\begin{aligned}
\rho(t)= & -i\left[H_{c}^{\prime}(t), \rho(t)\right] \\
& +\sum_{i j}\left\{k_{i j}(t)\left[2 a_{j} \rho(t) a_{i}^{\dagger}-a_{i}^{\dagger} a_{j} \rho(t)-\rho(t) a_{i}^{\dagger} a_{j}\right]\right. \\
& \left.+k_{i j}(t)\left[a_{i}^{\dagger} \rho(t) a_{j}+a_{j} \rho(t) a_{i}^{\dagger}-a_{i}^{\dagger} a_{j} \rho(t)-\rho(t) a_{j} a_{i}^{\dagger}\right]\right\}_{(8)}
\end{aligned}
$$

Here, $\rho(t)$ represents the attenuated density of the dark photons in the point break states, $H_{c}^{\prime}(t)=\sum_{i j} \omega_{c i j}^{\prime}(t) a_{i}^{\dagger} a_{j}$ represents the re-standardized Hamiltonian of the point break with respect to the point break frequencies $\omega_{c i i}^{\prime}(t)=\omega_{c i}^{\prime}(t)$, and the couplings of the dark photons among the point breaks $\omega_{c i j}^{\prime}(t)$ induced by the function. The factors $\kappa_{i j}(t)$ and $\tilde{\kappa}_{i j}(t)$ are characterized as the dark photonic dynamics in a PV semiconductor under extreme relativistic conditions. The re-standardized frequency, $\omega_{c i j}^{\prime}$, and the time-dependent factors, $\kappa_{i j}(t)$ and $\tilde{\kappa}_{i j}(t)$, are resolved purely via the non-perturbative principle. For a dark photon reservoir, the Hamiltonian is expressed as $H_{I}=\sum_{k} \lambda_{k} x q_{k}$, where $x$ and $q_{k}$ represent the locations of the primary unique point break and secondary unique point break reservoirs. Considering the quantum dynamics of a dark photon, for the whole reservoir within the point break, the Hamiltonian can be modified as $H_{I}=\sum_{k} V_{k}\left(a^{\dagger} b_{k}+b_{k}^{\dagger} a+a^{\dagger} b_{k}^{\dagger}+a b_{k}\right)$, which confirms the magnitude of the dark photonic dynamics within the point break. Therefore, the dark photonic dynamics can be characterized by the dissipated dark photon factors $\kappa(t)$ and $\tilde{\kappa}(t)$ (all the sub-indices $(i, j)$ are as in Eq. (2)). These factors can be calculated using the following exact unperturbed nonequilibrium functions [21, 22]:

$\omega_{c}^{\prime}(t)=-\operatorname{Im}\left[u\left(t, t_{0}\right) / u\left(t, t_{0}\right)\right]$

$k(t)=-\operatorname{Re}\left[u\left(t, t_{0}\right) / u\left(t, t_{0}\right)\right]$

$\hat{k}(t)=\dot{v}(t, t)+2 v(t, t) k(t)$.

where $u\left(t, t_{0}\right)$ represents the point break dark photon reservoir area and $v(t, t)$ represents the photon dynamics due to the induced reservoir. The latter is further clarified using the following integral-differential equation and non-equilibrium dynamics [23, 24]:

$\dot{u}\left(t, t_{0}\right)=-i \omega_{c} u\left(t, t_{0}\right)-\int_{t_{0}}^{t} \mathrm{~d} t^{\prime} g\left(t-t^{\prime}\right) u\left(t^{t}, t_{0}\right)$

$v(t, t)=\int_{t_{0}}^{t} \mathrm{~d} t \int_{0}^{t} \mathrm{~d} t_{2} u^{*}\left(t_{1}, t_{0}\right) \hat{g}\left(t_{1}-t_{2}\right) u\left(t_{2}, t_{0}\right)$

where $\mathrm{v}_{\mathrm{c}}$ represents the prime frequency at the point break. Therefore, the integral functions in Eqs. (12) and (13) determine the backup functions within the point breaks, and the number of dark photons created by the non-equilibrium state is expressed uniquely per unit area $J(\varepsilon)$ of the dark photonic structure using the following relations: $g\left(t-t^{\prime}\right)=\int \mathrm{d} \omega J(\omega) e^{-i \omega\left(t-t^{\prime}\right)}$ a nd $\quad \tilde{g}\left(t-t^{\prime}\right)=\int \mathrm{d} \omega J(\omega) \bar{n}(\omega, T) e^{-i \omega\left(t-t^{\prime}\right)}, \quad$ where $\bar{n}(\omega, T)=1 /\left[e^{\hbar \omega / k_{B} T}-1\right]$ represents the primary dark photon dynamics in the PV panel at a temperature $T$. The unit area $J(\omega)$ is defined in relation to the density of the states (DOS) $\rho(\omega)$ of the dark photon production in the semiconductor panel with a magnitude of $V_{k}$, between the point breaks and PV circuits,

$J(\omega)=\sum_{k}\left|V_{k}\right|^{2} \delta\left(\omega-\omega_{k}\right)=\rho(\omega)|V(\omega)|^{2}=[n * e(1+2 n)]^{4}$

Finally, I have summarized the dark photon production proliferation with respect to the dark photon dynamic state in the PV panel, such that $V_{k} \rightarrow V(\omega)$ and the $i$ of $V_{i k}$ in Eq. (1) can be counted at a single-diode mode point break. Thus, the non-equilibrium dark photon production $J(\omega)$, as 
Fig. 2 Single-diode model of a solar cell. It incorporates series and parallel circuit connections utilizing photonic energy (current), a diode, and two resistors

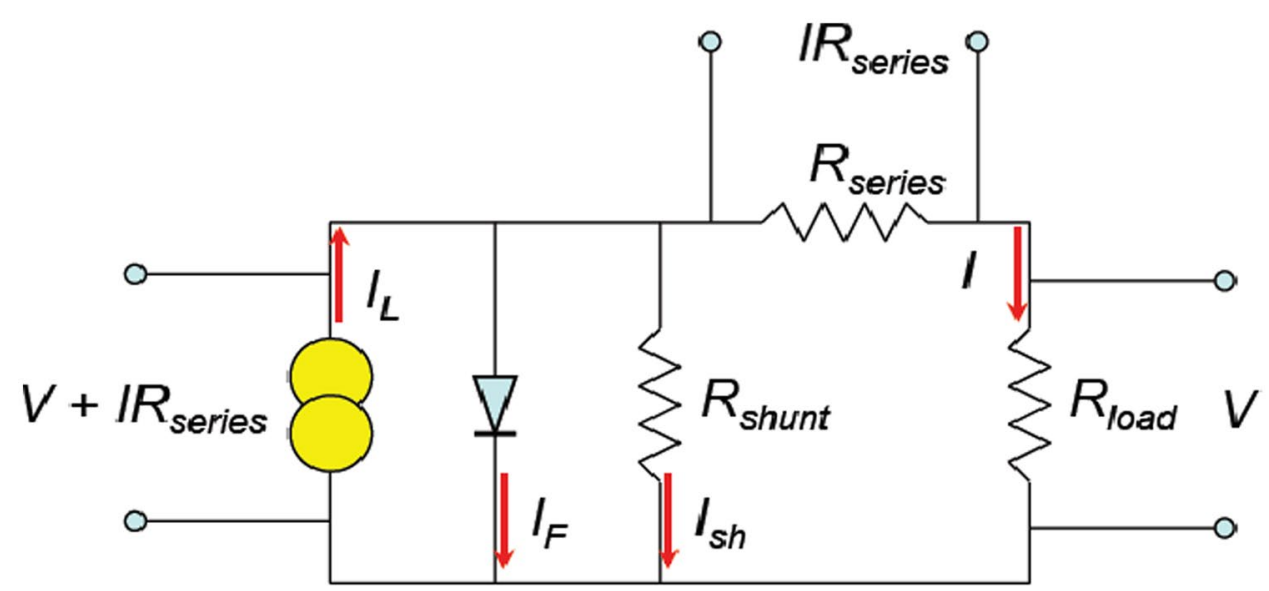

$$
I=I_{L}-\underbrace{I_{s}\left\{\exp \left[\frac{e\left(V+I R_{s}\right)}{m k_{B} T}\right]-1\right\}}_{I_{F}}-\underbrace{\frac{V+I R_{s}}{R_{s h}}}_{I_{s h}}
$$

considered in Eq. (14), can be obtained using the following simplified equation:

$J(\omega)=[n * e(1+2 n)]^{4}$

where $n=E=h f$ is the number of photons (i.e., the Bose-Einstein photon distribution) in the equilibrium stage and $e$ is the atmospheric constant and is 1 when there is a clear sky.

\section{Transformation of dark photon into energy}

To transform a dark photon into an HDP and then convert it into electricity, I have proposed using a semiconductor panel to perform ultrarelativistic reactions in the cell [25,
26]. Thus, a semiconductor module will be built by connecting solar cells in series and parallel, utilizing dark photon energy (current), a diode and two resistors; this system is called a solar cell single-diode model (Fig. 2).

The semiconductor model is thus described by the $I-V$ equation of PV cells for the single-diode mode. The $I-V$ relationship equation in the PV panel can be expressed as

$I=I_{L}-I_{O}\left\{\exp ^{\left[\frac{q\left(V+I_{R S}\right)}{A k T C}\right]}-1\right\}-\frac{\left(V+I_{\mathrm{RS}}\right)}{R_{\mathrm{Sh}}}$

$I_{L}$ represents the photon generating current, $I_{O}$ represents the saturated current in the diode, $R_{\mathrm{s}}$ represents the resistance in the series, $A$ represents the passive function of the

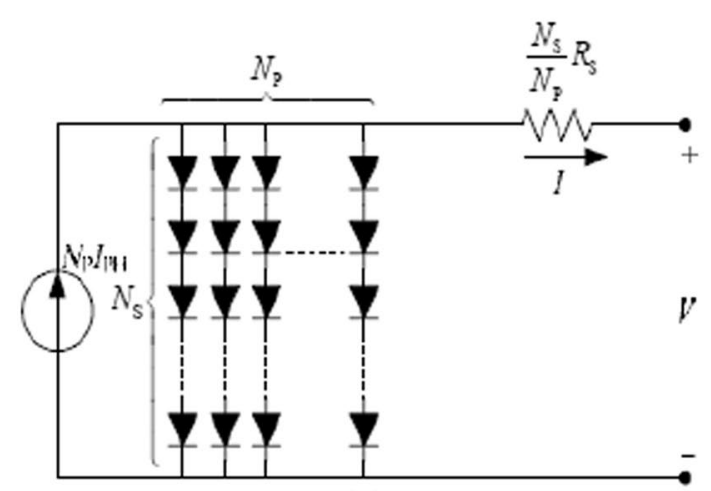

(a)



(b)

Fig. 3 Equivalent circuit models of the PV modules. a Normal and $\mathbf{b}$ normalized 
diode, $k\left(=1.38 \times 10^{-23} \mathrm{~W} / \mathrm{m}^{2} \mathrm{~K}\right)$ represents Boltzmann's constant, $q\left(=1.6 \times 10^{-19} \mathrm{C}\right)$ represents the magnitude of the charge of an electron and $T_{\mathrm{C}}$ represents the functional cell temperature. Subsequently, the $I-q$ relationship in the PV cells varies owing to the diode current and/or saturation current, which can be expressed as [27, 28]

$I_{O}=I_{\mathrm{RS}}\left(\frac{T_{C}}{T_{\text {ref }}}\right)^{3} \exp \left[\frac{q E G\left(\frac{1}{T_{\text {ref }}}-\frac{1}{T_{C}}\right)}{K A}\right]$

where $I_{\mathrm{RS}}$ represents the saturation current, considering the functional temperature and solar irradiance, and $q E G$ represents the band-gap energy moving into the silicon and graphene PV cell, considering the normal, and is normalized (Fig.3).

Considering the PV module, the $I-V$ equation, with the exception of the $I-V$ curve, is a conjunction of the $I-V$ curves of all the cells in the PV panel. Therefore, the equation can be rewritten as follows to determine the $V-R$ relationship:

$V=-\mathrm{IR}_{s}+K \log \left[\frac{I_{L}-I+I_{O}}{I_{O}}\right]$

Here, $K$ is as a constant $\left(=\frac{A k T}{q}\right)$, and $I_{\mathrm{mo}}$ and $V_{\mathrm{mo}}$ are the current and voltage in the PV panel. Therefore, the relationship between $I_{\mathrm{mo}}$ and $V_{\mathrm{mo}}$ is the same as the PV cell $I-V$ relationship:

$V_{\mathrm{mo}}=-I_{\mathrm{mo}} R_{S \mathrm{mo}}+K_{\mathrm{mo}} \log \left(\frac{I_{L \mathrm{mo}}-I_{\mathrm{mo}}+I_{O \mathrm{mo}}}{I_{O \mathrm{mo}}}\right)$

where $I_{L \mathrm{mo}}$ represents the photon-generated current, $I_{O \mathrm{mo}}$ represents the saturated current into the diode, $R_{s m o}$ represents the resistance in series and $K_{\mathrm{mo}}$ represents the factorial constant. Once all non-series (NS) cells are connected in series, the series resistance is counted as the summation

Table 1 MCMC calculations of the dark photon parameters assuming $N_{\text {eff }}$ of the relativistic neutrinos at the $95 \%$ confidence limit for the neutrino parameters

\begin{tabular}{lll}
\hline$\Omega_{b} h^{2}$ & $0.02229 \pm 0.00038$ & $0.02206 \pm 0.00081$ \\
$\Omega_{c} h^{2}$ & $0.1333 \pm 0.0086$ & $0.1313 \pm 0.0094$ \\
$\tau$ & $0.082 \pm 0.012$ & $0.083 \pm 0.014$ \\
$H_{0}$ & $74.3 \pm 2.2$ & $74.2 \pm 2.1$ \\
$n_{s}$ & $0.977 \pm 0.011$ & $0.972 \pm 0.021$ \\
$\log \left(10^{10} A_{\mathrm{s}}\right)$ & $3.195 \pm 0.035$ & $3.196 \pm 0.035$ \\
$A_{\mathrm{SZ}}$ & $<1.2$ & $<1.4$ \\
$A_{\mathrm{C}}\left[\mu K^{2}\right]$ & $<14.3$ & $<14.6$ \\
$A_{\mathrm{P}}\left[\mu K^{2}\right]$ & $<25.2$ & $<24.7$ \\
$N_{\text {eff }}$ & $4.08_{-0.18-0.68}^{+0.18+0.71}$ & $3.89_{-0.19-0.70}^{+0.19+0.70}$ \\
$c_{\text {eff }}^{2}$ & $1 / 3$ & $0.312_{-0.007-0.026}^{+0.008+0.026}$ \\
$c_{\text {vis }}^{2}$ & $1 / 3$ & $0.29_{-0.06-0.16}^{+0.04+0.21}$ \\
$X_{\text {min }}^{2}$ & & 7591.5 \\
\hline
\end{tabular}

of the resistance of each cell in the series $R_{s m o}=N_{S} \times R_{S}$, and the constant factor can be expressed as $K_{\text {mo }}=N_{S} \times K$. There is a certain amount of current flowing into the series of connected cells; thus, the current flow in Eq. 5 remains the same for each component, i.e., $I_{o \text { mo }}=I_{o}$ and $I_{L \mathrm{mo}}=I_{L}$. Thus, the module $I_{\mathrm{mo}}-V_{\mathrm{mo}}$ equation for the $N_{S}$ series of connected cells is written as

$V_{\mathrm{mo}}=-I_{\mathrm{mo}} N_{\mathrm{S}} R_{\mathrm{S}}+N_{\mathrm{S}} K \log \left(\frac{I_{L}-I_{\mathrm{mo}}+I_{o}}{I_{o}}\right)$

Similarly, the current-voltage calculation can be rewritten for the parallel connections once all $N_{\mathrm{P}}$ cells are connected in parallel and can be expressed as follows [29, 30]:

$V_{\mathrm{mo}}=-I_{\mathrm{mo}} \frac{R_{\mathrm{s}}}{N_{\mathrm{p}}}+K \log \left(\frac{N_{\mathrm{sh}} I_{L}-I_{\mathrm{mo}}+N_{\mathrm{p}} I_{o}}{N_{\mathrm{p}} I_{o}}\right)$

Because the photon-generated current will depend primarily on the solar irradiance and relativistic temperature conditions of the PV panel, the current can be calculated using the following equation:

$I_{L}=G\left[I_{\mathrm{SC}}+K_{I}\left(T_{C}-T_{\text {ref }}\right)\right] * V_{\mathrm{mo}}$

where $I_{\mathrm{sc}}$ represents the PV current at $25^{\circ} \mathrm{C}$ and $\mathrm{kW} / \mathrm{m}^{2}, K_{I}$ represents the relativistic $\mathrm{PV}$ panel coefficient, $T_{\text {ref }}$ represents the PV panel's functional temperature, and $G$ represents the solar energy in $\mathrm{kW} / \mathrm{m}^{2}$ (Table 1 ).

Table 2 The degeneracy among the parameters $N_{v}^{s}, c_{\text {eff }}^{2}$, and $c_{\text {vis }}^{2}$ is shown by plotting the $2 \mathrm{D}$ contours, which suggest that a degeneracy is present between $c_{\text {eff }}^{2}$ and $N_{v}^{s}$

\begin{tabular}{lll}
\hline Model & $\begin{array}{l}\text { Varying } c_{\text {eff }}^{2}, c_{\text {vis }}^{2} \\
(A)\end{array}$ & $\begin{array}{l}c_{\text {eff }}^{2}=1 / 3, \text { varying } c_{\text {vis }}^{2} \\
(B)\end{array}$ \\
\hline$\Omega_{b} h^{2}$ & $0.02177 \pm 0.00066$ & $0.02206 \pm 0.00081$ \\
$\Omega_{c} h^{2}$ & $0.135 \pm 0.010$ & $0.1313 \pm 0.0094$ \\
$\tau$ & $0.086 \pm 0.013$ & $0.083 \pm 0.014$ \\
$H_{0}$ & $72.8 \pm 2.1$ & $74.2 \pm 2.1$ \\
$n_{s}$ & $0.989 \pm 0.014$ & $0.972 \pm 0.021$ \\
$\log \left(10^{10} A_{\mathrm{s}}\right)$ & $3.178 \pm 0.035$ & $3.196 \pm 0.035$ \\
$A_{\mathrm{SZ}}$ & $<1.6$ & $<1.4$ \\
$A_{\mathrm{C}}\left[\mu K^{2}\right]$ & $<15.0$ & $<14.6$ \\
$A_{\mathrm{P}}\left[\mu K^{2}\right]$ & $<24.8$ & $<24.7$ \\
$N_{\text {eff }}$ & $1.10_{-0.23-0.72}^{+0.19+0.79}$ & $1.46_{-0.21-0.74}^{+0.21+0.76}$ \\
$c_{\text {eff }}^{2}$ & $0.24_{-0.02-0.13}^{+0.03+0.08}$ & $1 / 3$ \\
$c_{\text {vis }}^{2}$ & $<0.91$ & $<0.74$ \\
$X_{\text {min }}^{2}$ & 7590.5 & 7592.0 \\
\hline
\end{tabular}

The models with lower values of $N_{v}^{s}$ are more compatible with $c_{\text {eff }}^{2}=0$ since the effect of $c_{\text {eff }}^{2}$ on the CMB spectrum is smaller. No apparent degeneracy is present between $c_{\mathrm{vis}}^{2}$ and the remaining parameters, since $c_{\text {vis }}^{2}$ is weakly constrained for the neutrino parameters by the current data at the $95 \%$ confidence level 


\section{Results and discussion}

\section{Dark photon modeling}

The relativistic contributions of $N_{\text {eff }}$ and its corresponding perturbation parameters $c_{\text {vis }}^{2}$ and $c_{\text {eff }}^{2}$ are related to the standard neutrinos which is written as $N_{v}^{S}=N_{\text {eff }}$ and was determined from the variables $c_{\text {vis }}^{2}$ and $c_{\text {eff }}^{2}$. The constraints on the dark photon parameters of varying $N_{\text {eff }}$, with and without variations in their perturbations, depend on two functions of WMAP7, ACT, SPT, DR7, H0 analysis [31, 32]. Therefore, first I have run an analysis to fix the perturbation parameters to match the standard values, i.e., $c_{\text {eff }}^{2}=c_{\text {vis }}^{2}=1 / 3$. The WMAP7 $+\mathrm{ACT}+\mathrm{SPT}+\mathrm{DR} 7+\mathrm{H} 0$ calculation reveals that the dark photon $N_{\text {eff }}=4.08_{-0.68}^{+0.71}$ at $95 \% \mathrm{CI}$, which is very much feasible when considering the variations in the perturbation parameters (right column) once the constraints are somewhat shifted towards smaller values, with $N_{\text {eff }}=3.89_{-0.70}^{+0.70}[33,34]$. Consequently, the constraint $c_{\text {eff }}^{2}=0.312 \pm 0.026$ is fully consistent with the expectations of a free streaming component and the anisotropies in the neutrinos at a statistical significance with $c_{\mathrm{vis}}^{2}=0.29_{-0.16}^{+0.21}[35,36]$.

As the results in Table 2 show, the evidence for an extra background is strong, with $N_{v}^{s}=1.46_{-0.74}^{+0.76}$ at the $95 \% \mathrm{CI}$, when only the variations in the $c_{\text {vis }}^{2}$ component are considered, but the constraint is $N_{v}^{s}=1.10_{-0.72}^{+0.79}$ when variations in $c_{\text {eff }}^{2}$ are also considered. Again, the data provide a clear determination of $c_{\text {eff }}^{2}$, such that $c_{\text {eff }}^{2}=0.24_{-0.13}^{+0.08}$ at the $95 \%$ $\mathrm{CI}$, which is in reasonable agreement with the standard $c_{\text {eff }}^{2}=1 / 3$ value, while the significant constraint of the dark photons is obtained from $c_{\text {vis }}^{2}$

Consequently, the model-independent analysis of the extra relativistic degrees of freedom for dark photons has been analyzed by considering the maximum likelihood value $L$ as a function of $N_{\text {eff }}$ and by considering $\ln \left(L N_{\text {eff }} / L_{\text {max }}\right)$ as a function of $N_{\text {eff }}$. Where $L_{\max }$ has a maximum likelihood across the entire chains, the number of relativistic degrees of freedom $N_{\text {eff }}$ varies given that $c_{\text {eff }}^{2}=c_{\text {vis }}^{2}=1 / 3$, which clearly indicates the presence of dark radiation $N_{\text {eff }}$ with a $\Delta \chi^{2}=14.56$.

\section{Dark photon transformation}

To mathematically calculate the transformation of an HDP in the semiconductor panel, I initially solved for the dynamic dark photon by integrating Eqs. (7) and (8). It is well established that, owing to the spatially variable condition $J(\omega)$, the semiconductor panels produce different dark photon dynamics [37]. The unit area $J(\omega)$ has a persistent weakcoupling limit, and the Weisskopf-Wigner approximation rule and/or the Markovian master equation is equal to the dark photon activation. Consequently, all HDP photon activations will have dynamic dark photon state modes $(1 \mathrm{D}, 2 \mathrm{D}$, 3D) in the semiconductor cell, which can be expressed as described in Table 3 [24, 38].

Subsequently, a fine high-level frequency cut-off of $\Omega_{\mathrm{C}}$ is employed to avoid the bifurcation of the DOS in a 3D semiconductor cell. Similarly, a sharpended high-level frequency cut-off has been employed at $\Omega_{\mathrm{d}}$ and maintain it into a positive DOS in 2D and 1D semiconductor cells. Hence, $\mathrm{Li}_{2}(x)$ acts as a dilogarithm variable and $e_{\mathrm{rfc}}(x)$ acts as an additive variable. Subsequently, the DOS of the various semiconductor cells, denoted as $\rho_{\mathrm{PC}}(\omega)$, is determined by calculating the dark photon eigenfrequencies and eigenfunctions of Maxwell's rules, while considering the nanostructures of the semiconductor [3, 27, 29]. For a 1D semiconductor cell, the corresponding DOS is denoted as $\rho_{\mathrm{PC}}(\omega) \propto \frac{1}{\sqrt{\omega-\omega_{e}}} \Theta\left(\omega-\omega_{e}\right)$, where $\Theta\left(\omega-\omega_{e}\right)$ represents the Heaviside step function and $e$ in $\omega_{e}$ represents the frequency in the PBE considering the DOS.

The dark photonic modes depict the crossover of the 1D and 2D semiconductor cells into PV cells, and a complex transitional state of the 3D cell into the semiconductor cell
Table 3 The dark photonic structures in the different DOS dimensional modes in the PV cell

\begin{tabular}{lll}
\hline $\begin{array}{l}\text { Photo vol- } \\
\text { taic }(\mathrm{PV})\end{array}$ & Unit area $J(\omega)$ for different DOS & Reservoir-induced self-energy correction $\Sigma(\omega)$ \\
\hline $1 \mathrm{D}$ & $\frac{C}{\pi} \frac{1}{\sqrt{\omega-\omega_{e}}} \Theta\left(\omega-\omega_{e}\right)$ & $-\frac{C}{\sqrt{\omega_{e}-\omega}}$ \\
$2 \mathrm{D}$ & $-\eta\left[\ln \left|\frac{\omega-\omega_{0}}{\omega_{0}}\right|-1\right] \Theta\left(\omega-\omega_{e}\right) \Theta\left(\Omega_{d}-\omega\right)$ & $\eta\left[\operatorname{Li}_{2}\left(\frac{\Omega_{d}-\omega_{0}}{\omega-\omega_{0}}\right)-\mathrm{Li}_{2}\left(\frac{\omega_{0}-\omega_{e}}{\omega_{0}-\omega}\right)-\ln \frac{\omega_{0}-\omega_{e}}{\Omega_{d}-\omega_{0}} \ln \frac{\omega_{e}-\omega}{\omega_{0}-\omega}\right]$ \\
$3 \mathrm{D}$ & $\chi \sqrt{\frac{\omega-\omega_{e}}{\Omega_{C}}} \exp \left(-\frac{\omega-\omega_{e}}{\Omega_{C}}\right) \Theta\left(\omega-\omega_{e}\right)$ & $\chi\left[\pi \sqrt{\frac{\omega_{e}-\omega}{\Omega_{C}}} \exp \left(-\frac{\omega-\omega_{e}}{\Omega_{C}}\right) \operatorname{erf} c \sqrt{\frac{\omega_{e}-\omega}{\Omega_{C}}}-\sqrt{\pi}\right]$ \\
\hline
\end{tabular}

These structures correspond to different unit areas $J(\omega)$ and self-induced energies in the reservoir $\Sigma(\omega)$, which were determined by the photon dynamics in the extreme relativistic semiconductor cell. The variables $C$, $\eta$ and $\chi$ function like coupled forces between the point breaks and semiconductors in 1D, 2D, and $3 \mathrm{D}$, oriented into the semiconductor cell 
from a PBG area to a dark photonic band (PB) area $\left(\partial / \omega_{e}\right)$. [27]. The DOS is, therefore, calculated to conduct the $3 \mathrm{D}$ isotropic analysis in the PV cells to predict the error-free qualitative states of the non-Weisskopf-Wigner modes and the dark photon-photon collision states in the semiconductor cells $[14,24,39]$. Therefore, for a 3D semiconductor cell, a DOS close to the PBE is implemented via an anisotropic DOS, $\varrho_{\mathrm{PC}}(\omega) \propto \frac{1}{\sqrt{\omega-\omega_{e}}} \Theta\left(\omega-\omega_{e}\right)$, which is then clarified with respect to the electromagnetic field (EMF) vector [2, 28]. For the 2D and 1D semiconductor cells, the photon DOS exhibits a purely logarithm divergence close to the PBE, which is approximated as $\varrho_{\mathrm{PC}}(\omega) \propto-\left[\ln \left|\left(\omega-\omega_{0}\right) / \omega_{0}\right|-1\right] \Theta\left(\omega-\omega_{e}\right)$, where $\omega_{e}$ represents the central point of the peak logarithm. The unit area $J(\omega)$ is defined as the production field of the DOS in the semiconductor cell, delineated by the fine dark photonic magnitude $V(\omega)$ within the PB and PV cell [30, 40],

$J(\omega)=\varrho(\omega)|V(\omega)|^{2}$



Fig. 4 The dark photonic band structure and mode of energy conversion. a Unit area for various DOS values for 1D, 2D and 3D semiconductor cells $J(\omega)$. b Dark photonic mode of the frequencies for functional tuning $\left(\partial / \omega_{e}\right)$. $\mathbf{c}$ Dark photonic mode of the magnitude of energy released, calculated using Eq. (2)
Hereafter, I consider the PB frequency $\omega_{c}$ and proliferative dark photon dynamics using the function $u\left(t, t_{0}\right)$ for the photon structure in the equation $a(t)=u\left(t, t_{0}\right) a\left(t_{0}\right)$. It is calculated using the dissipative integro-differential equation given in Eq. (25) and is expressed as

$u\left(t, t_{0}\right)=\frac{1}{1-\Sigma^{\prime}\left(\omega_{b}\right)} e^{-i \omega\left(t-t_{0}\right)}+\int_{\omega_{e}}^{\infty} \mathrm{d} \omega \frac{J(\omega) e^{-i \omega\left(t-t_{0}\right)}}{\left[\omega-\omega_{c}-\Delta(\omega)\right]^{2}+\pi^{2} J^{2}(\omega)}$

where $\Sigma^{\prime}\left(\omega_{b}\right)=[\partial \Sigma(\omega) / \partial \omega]_{\omega=\omega_{b}}$ and $\Sigma(\omega)$ represents the reservoir-induced PB photon self-energy correction

$\Sigma(\omega)=\int_{\omega_{e}}^{\infty} \mathrm{d} \omega^{\prime} \frac{J\left(\omega^{\prime}\right)}{\omega-\omega^{\prime}}$

Here, the frequency $\omega_{b}$ in Eq. (2) represents the dark photonic frequency mode in the PBG $\left(0<\omega_{b}<\omega_{e}\right)$ and is calculated using the pole condition $\omega_{b}-\omega_{c}-\Delta\left(\omega_{b}\right)=0$, where $\lesssim \Delta(\omega)=\mathcal{P}\left[\int \mathrm{d} \omega^{\prime} \frac{J\left(\omega^{\prime}\right)}{\omega-\omega^{\prime}}\right]$ is a principal-value integral.

Furthermore, the detailed dark photonic dynamics, given the proliferation magnitude $\left|u\left(t, t_{0}\right)\right|$, have been cal-
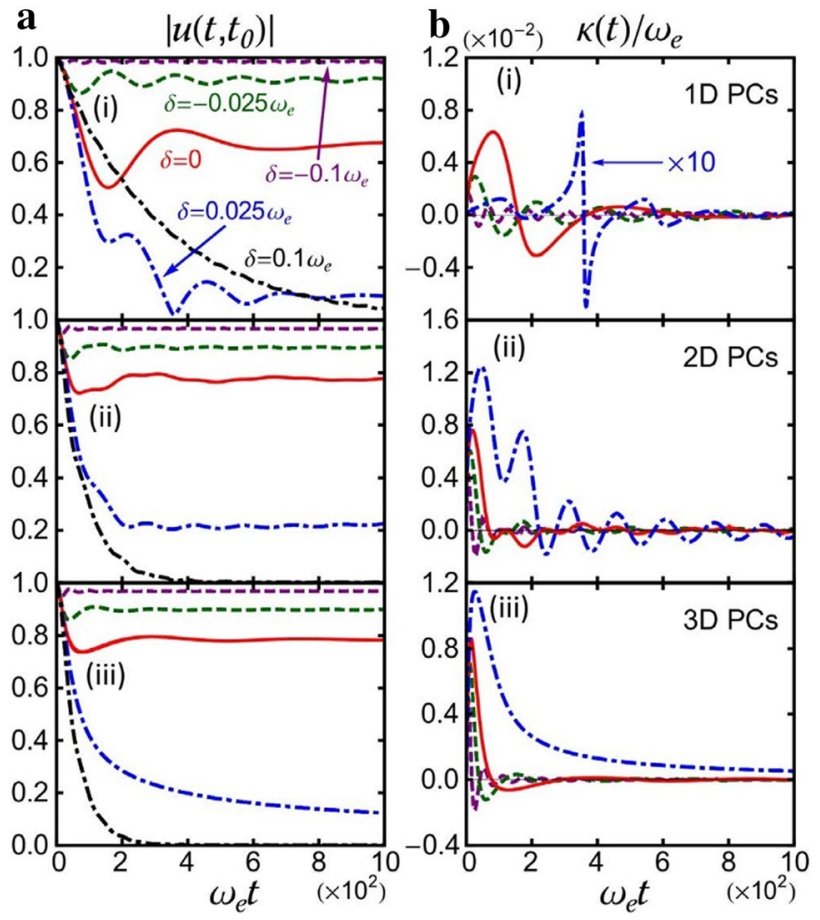

Fig. 5 Proliferation of the dynamic dark photons in the semiconductor cells. a Given the PB area $\langle a(t)\rangle=5 u\left(t, t_{0}\right)\left\langle a\left(t_{0}\right)\right\rangle$, and $\mathbf{b}$ the dynamic dark photonic rate $k(t)$, plotted for the (i) $1 \mathrm{D}$, (ii) $2 \mathrm{D}$ and (iii) $3 \mathrm{D}$ semiconductor cells $[27,28]$ 




(a)

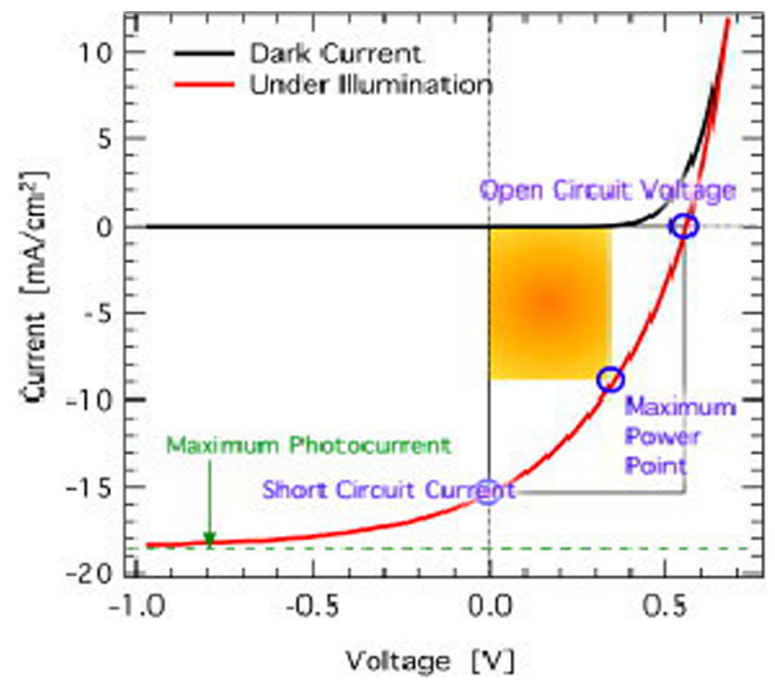

(b)

Fig. 6 Solar cell current-voltage characteristic features for the conceptual function of $\mathbf{a}$ an optimal working point for current production and $\mathbf{b}$ the current-voltage module of the current source near the short-circuit point as a voltage source in the vicinity of the open circuit point

culated and are shown in Fig. 4a for the 1D, 2D and 3D semiconductor cells with respect to various detuning $\delta$, integrated from the PBG area to the PB area [26, 29, 36]. The dark photonic dynamic rate $\kappa(t)$ is shown in Fig. 4b, neglecting the function $\delta=0.1 \omega_{e}$. The calculated result indicates that dynamic dark photons are produced at a higher rate once $\omega_{c}$ crosses from the PBG to the PB area. Because the range of $u\left(t, t_{0}\right)$ is $1 \geq\left|u\left(t, t_{0}\right)\right| \geq 0$, I have defined the crossover area as being related to the condition $0.9 \gtrsim\left|u\left(t \rightarrow \infty, t_{0}\right)\right| \geq 0$. This corresponds to $-0.025 \omega_{e} \lesssim \delta \lesssim 0.025 \omega_{e}$, with an activation rate $\kappa(t)$ within the PBG $\left(\delta<-0.025 \omega_{e}\right)$ and in the vicinity of the PBE $\left(-0.025 \omega_{e} \lesssim \delta \lesssim 0.025 \omega_{e}\right)$ (Fig. 5).

To convert the dark photons into HDPs, I have first considered the $\mathrm{PB}$ as the Fock state photon number $n_{0}$, i.e. $\rho\left(t_{0}\right)=\left|n_{0} n_{0}\right|$, which is obtained theoretically through the real-time quantum feedback control [34] and then by solving Eq. (2), with consideration for the state of the dark photon activation at time $t$ :

$$
\begin{aligned}
& \rho(t)=\sum_{n=0}^{\infty} \mathcal{P}_{n}^{\left(n_{0}\right)}(t)\left|n_{0} n_{0}\right| \\
& \mathcal{P}_{n}^{\left(n_{0}\right)}(t)=\frac{[v(t, t)]^{n}}{[1+v(t, t)]^{n+1}}[1-\Omega(t)]^{n_{0}} \\
&= \sum_{k=0}^{\min \left\{n_{0}, n\right\}}\left(\begin{array}{c}
n_{0} \\
k
\end{array}\right)\left(\begin{array}{l}
n \\
k
\end{array}\right)\left[\frac{1}{v(t, t)} \frac{\Omega(t)}{1-\Omega(t)}\right]^{k}
\end{aligned}
$$

where $\Omega(t)=\frac{\left|u\left(t, t_{0}\right)\right|^{2}}{1+v(t, t)}$. Therefore, the results suggest that a Fock state photon will evolve into a different Fock state of $\mid n_{0} i s \mathcal{P}_{n}^{\left(n_{0}\right)}(t)$. The activation of the dark photon dissipation $\mathcal{P}_{n}^{\left(n_{0}\right)}(t)$ in the primary state $\mid n_{0}=5$ and the steady-state limit, $\mathcal{P}_{n}^{\left(n_{0}\right)}(t \rightarrow \infty)$, are shown in Fig. 6. Therefore, the activation of the dark photon will ultimately reach a thermal non-equilibrium state, with the dark photonic structure written as:

$\mathcal{P}_{n}^{\left(n_{0}\right)}(t \rightarrow \infty)=\frac{\left[\bar{n}\left(\omega_{c}, T\right)\right]^{n}}{\left[1+\bar{n}\left(\omega_{c}, T\right)\right]^{n+1}}$

To probe HDP production, I have further proposed to analyze the coherent states of the dark photons by solving Eq. (2) for the activation state of the dark photons, which can be expressed as

$\rho(t)=\mathcal{D}[\alpha(t)] \rho_{T}[v(t, t)] \mathcal{D}^{-1}[\alpha(t)]$

where $\mathcal{D}[\alpha(t)]=\exp \left\{\alpha(t) \alpha^{\dagger}-\alpha^{*}(t) \alpha\right\}$ represents the displacement driver with $\alpha(t)=u\left(t, t_{0}\right) \alpha_{0}$ and

$\rho_{T}[v(t, t)]=\sum_{n=0}^{\infty} \frac{\left[v(t, t)^{n}\right]}{[1+v(t, t)]^{n+1}}|n n|$

Here $\rho_{T}$ represents a thermal state with a mean particle number $v(t, t)$, and Eq. (17) reveals that the primary breakpoint of the cavity state will evolve into a displaced thermal state $[37,41]$, which is a mixture of the displaced numerical states $\mathcal{D}[\alpha(t)] \mid n^{37}$. Thus, the dark photon activation representation, Eq. (29), can be written as 


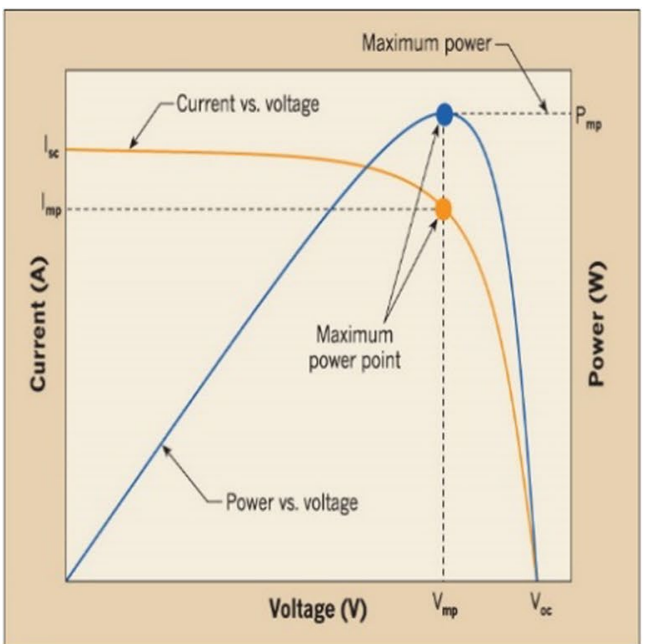

(a)

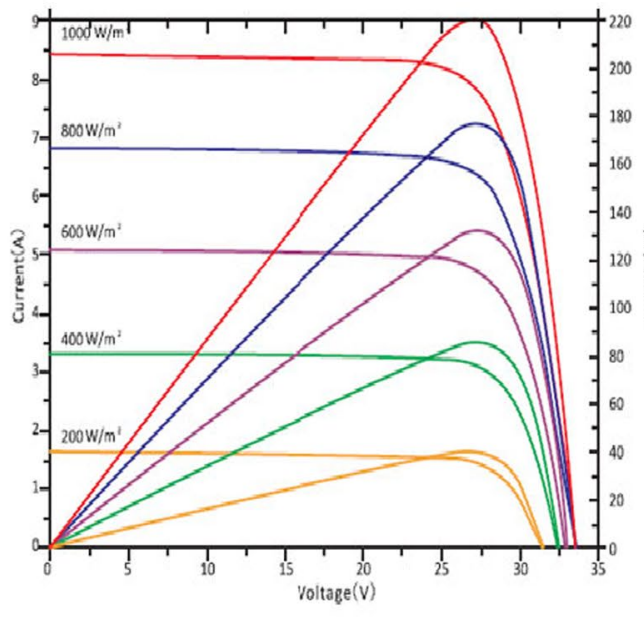

(b)



.
Fig. $7 \quad I-V$ characteristics of the PV module at various temperatures and a constant solar irradiance. a Current-voltage-power simulation considering the maximum power point tracking, and $\mathbf{b}$ the simulation

$$
\begin{aligned}
m|\rho(t)| n & =J(\omega)=e^{-\Omega(t)\left|\alpha_{0}\right|^{2}} \frac{[\alpha(t)]^{m}\left[\alpha^{*}(t)\right]^{n}}{[1+v(t, t)]^{m+n+1}} \\
& =\sum_{k=0}^{\min \{m, n\}} \frac{\sqrt{m ! n !}}{(m-k) !(n-k) ! k !}\left[\frac{v(t, t)}{\Omega(t)\left|\alpha_{0}\right|^{2}}\right]^{k} \\
& =[n * e(1+2 n)]
\end{aligned}
$$

where the activation of an HDP in the semiconductor panel $(m|\rho(t)| n)$ shall indeed evolve into an extreme relativistic thermal state $[1+v(t, t)]^{m+n+1}$ with the non-equilibrium condition $[\alpha(t)]^{m}\left[\alpha^{*}(t)\right]^{n}$. Simply, it can be said that HDP production in semiconductor panels is viable with the implementation of Higgs boson quantum collisions under extreme relativistic conditions and produces energy.

\section{Transform dark photon into energy}

To convert an HDP into electricity under extreme relativistic conditions, a single-diode semiconductor panel consisting of a small disk semiconductor attached via wire to a circuit consisting of a positive and a negative film of silicon placed under a thin slice of glass and attached to graphene has been used. The semiconductor panel must have an open circuit point such that the current and voltage are at a maximum and an open circuit voltage $V_{\text {oc }}$. In addition, the maximum power point can be determined using the maximum current and voltage calculation immediately after capturing a HDP $[38,41]$. The power delivered by a semiconductor PV panel will thus have the capability to reach a maximum value at the points $\left(I_{\mathrm{mp}}, V_{\mathrm{mp}}\right)[36,40]$. To confirm the semiconductor results for the $I-V$ and $\mathrm{P}-\mathrm{V}$ characteristics at varying solar irradiances $\left(200-1000 \mathrm{~W} / \mathrm{mm}^{2}\right)$ in $200 \mathrm{~W} / \mathrm{mm}^{2}$ increments, with a constant module temperature $\left(25^{\circ} \mathrm{C}\right)$

panel's capability of such current-voltage flow, the optimal working points and the dark functionality under the illuminating state of the semiconductor panel has been analyzed.

To determine the results of the $I-V$ and $\mathrm{P}-\mathrm{V}$ features of the PV panel for the relativistic temperatures from 25 to $45{ }^{\circ} \mathrm{C}$ using $5{ }^{\circ} \mathrm{C}$ increments and a constant solar irradiance $800 \mathrm{~W} / \mathrm{nm}^{2}$, the short-circuit current $\left(I_{\mathrm{sc}}\right)$ constant is considered at constant level. Simply, once the temperature increases due to the relativistic temperature, both the open circuit voltage $\left(V_{\mathrm{oc}}\right)$ and maximum power output of the $\mathrm{P}-\mathrm{V}$ module increase in the semiconductor panel, and the result will be a non-linear flow of electrical energy in the $\mathrm{P}-\mathrm{V}$ module (Fig. 7).

\section{Conclusions}

Dark photon is modeled by implementing a Higgs boson quantum collision under extreme relativistic conditions to transform it into an activated photon (HDP) to create energy. Thus, a series of mathematical calculations (dark photon modeling, dark photon transformation, dark photon conversion into energy) have been performed using MATLAB software that suggest that the transformation of dark photons into HDP by Higgs boson [BR $\left(\mathrm{H} \rightarrow \gamma \gamma^{-}\right.$] particle collisions under extreme relativistic conditions (ERC) is quite feasible. Simply, it can be said that the HDP formation from a dark photon is just a high-energy photo-physical reaction that activates the dark photon into an energy level photon, which would be a new field of energy science. Indeed, it will 
open a new door in science for better understanding of this hidden power.

Acknowledgements This research was supported by Green Globe Technology under Grant RD-02017-02 for building a better environment. Any findings, predictions, and conclusions described in this article are solely performed by the authors and we confirm that there is no conflict of interest for publishing in a suitable journal.

Open Access This article is distributed under the terms of the Creative Commons Attribution 4.0 International License (http://creativeco mmons.org/licenses/by/4.0/), which permits unrestricted use, distribution, and reproduction in any medium, provided you give appropriate credit to the original author(s) and the source, provide a link to the Creative Commons license, and indicate if changes were made.

\section{References}

1. Gupta, N., Singh, S.P., Dubey, S.P., Palwalia, D.K.: Fuzzy logic controlled three-phase three-wired shunt active power filter for power quality improvement. Int. Rev. Electr. Eng. (IREE) 6(3), 1118-1129 (2011)

2. Hossain, F.: Solar energy integration into advanced building design for meeting energy demand and environment problem. J. Energy Res. 17, 49-55 (2016)

3. Celik, A.N., Acikgoz, N.: Modelling and experimental verification of the operating current of mono-crystalline photovoltaic modules using four- and five-parameter models. Appl. Energy 84(1), 1-15 (2007)

4. Robyns, B., Francois, B., Degobert, P., Hautier, J.P.: Vector Control of Induction Machines. Springer, London (2012)

5. Douglas, J.S., Habibian, H., Hung, C.-L., Gorshkov, A.V., Kimble, H.J., Chang, D.E.: Quantum many-body models with cold atoms coupled to photonic crystals. Nat. Photonics 9, 326-331 (2015)

6. Boukhezzar, B., Siguerdidjane, H.: Nonlinear control with wind estimation of a DFIG variable speed wind turbine for power capture optimization. Energy Convers. Manag. 50(4), 885-892 (2009)

7. Xiao, W., Dunford, W.G., Capal, A.: A novel modeling method for photovoltaic cells. In: 35th Annul IEEE Power Electronics Specialists Conference, vol. 3, pp. 1950-1956. Aachen, Germany (2004)

8. Tan, Y.T., Kirschen, D.S., Jenkins, N.: A model of PV generation suitable for stability analysis. IEEE Trans. Energy Convers. 19(4), 748-755 (2004)

9. Zhu, Y., Hu, X., Yang, H., Gong, Q.: On-chip plasmon-induced transparency based on plasmonic coupled nanocavities. Sci. Rep. 46, 246-251 (2014)

10. Reinhard, A.: Strongly correlated photons on a chip. Nat. Photonics 6, 93-96 (2011)

11. Belkacem, H.G., Feinberg, B., Bossingham, R., Meyerhof, W.E.: Semiclassical dynamics and relaxation. Phys. Rev. Lett. 71, 1514 (1993)

12. Faida, H., Saadi, J.: Modelling, control strategy of DFIG in a wind energy system and feasibility study of a wind farm in Morocco. Int. Rev. Modell. Simul. (IREMOS) 3(6), 1350-1362 (2010)

13. Park, J., Kim, H., Cho, Y., Shin, C.: Simple modeling and simulation of photovoltaic panels using matlab/simulink. Adv. Sci. Technol. Lett. 73(FGCN 2014), 147-155 (2014)

14. Yang, L., Wang, S., Zeng, Q., Zhang, Z., Pei, T., Li, Y., Peng, L.-M.: Efficient photovoltage multiplication in carbon nanotubes. Nat. Photonics 5, 672-676 (2011)
15. Benavides, N.D., Chapman, P.L.: Modeling the effect of voltage ripple on the power output of photovoltaic modules. IEEE Trans. Ind. Electron. 55(7), 2638-2643 (2008)

16. Valluri, S.R., Becker, U., Grun, N., Scheid, W.: Relativistic collisions of highly-charged ions. J. Phys. B At. Mol. Phys. 17, 4359 (1984)

17. Gould, R.J.: Pair production in photon-photon collisions. Phys. Rev. 155, 1404-1407 (1967)

18. Gopal, C., Mohanraj, M., Chandramohan, P., Chandrasekar, P.: Renewable energy source water pumping systems-a literature review. Renew. Sustain. Energy Rev. (2013). https://doi. org/10.1016/j.rser.2013.04.012

19. Baur, G., Hencken, K., Trautmann, D.: Revisiting unitarity corrections for electromagnetic processes in collisions of relativistic nuclei. Phys. Rep. 453, 1 (2007)

20. Baur, G., Hencken, K., Trautmann, D., Sadovsky, S., Kharlov, Y.: Dense laser-driven electron sheets as relativistic mirrors for coherent production of brilliant X-ray and $\gamma$-ray beams. Phys. Rep. 364, 359 (2002)

21. Ghennam, T., Berkouk, E.M., Francois, B.: A vector hysteresis current control applied on three-level inverter. Application to the active and reactive power control of doubly fed induction generator based wind turbine. Int. Rev. Electr. Eng. (IREE) 2(2), 250-259 (2007)

22. Arnold, P.: Photon emission from ultrarelativistic plasmas. J. High Energy Phys. 33, 310-315 (2001)

23. Eichler, J., Stöhlker, T.: Radiative electron capture in relativistic ion-atom collisions and the photoelectric effect in hydrogen-like high-Z systems. Phys. Rep. 439, 1 (2007)

24. Hencken, K.: Transverse momentum distribution of vector mesons produced in ultraperipheral relativistic heavy ion collisions. Phys. Rev. Lett. 96(1), 012303 (2006)

25. Soon, J.J., Low, K.-S.: Optimizing photovoltaic model parameters for simulation. In: IEEE International Symposium on Industrial Electronics (2012)

26. Li, Q., Xu, D.Z., Cai, C.Y., Sun, C.P.: Recoil effects of a motional scatterer on single-photon scattering in one dimension. Sci. Rep. 64, 561-565 (2013)

27. Lo, P.-Y., Xiong, H.-N., Zhang, W.-M.: Breakdown of BoseEinstein distribution in photonic, crystals. Sci. Rep. 39, 631-637 (2015)

28. Artemyev, N., Jentschura, U.D., Serbo, V.G., Surzhykov, A.: Strong electromagnetic field EFFECTS in ultra-relativistic heavyion collisions eur. Phys. J. C 72, 1935 (2012)

29. Archidiacono, M.: Case for dark radiation. Phys. Rev. D 84, 123 $127(2011)$

30. Güçlü, M.C., Lib, J., Umarb, A.S., Ernstb, D.J., Strayer, M.R.: Electromagnetic lepton pair production in relativistic heavy-ion collisions. Ann. Phys. 272, 7 (1999)

31. Najjari, A., Voitkiv, A.B., Artemyev, A., Surzhykov, A.: Simultaneous electron capture and bound-free pair production in relativistic collisions of heavy nuclei with atoms. Phys. Rev. A 80, 012701 (2009)

32. Sharma, K.G., Bhargava, A., Gajrani, K.: Stability analysis of DFIG based wind turbines connected to electric grid. Int. Rev. Modell. Simul. (2013)

33. Klein, S.A.: Calculation of flat-plate collector loss coefficients. Solar Energy 17, 79-80 (1975)

34. Soedibyo, A., Pamuji, F.A., Mochamad, A.: Grid quality hybrid power system control of microhydro, wind turbine and fuel cell using fuzzy logic. Int. Rev. Modell. Simul. 93, 451-459 (2013)

35. Valluri, S.R., Becker, U., Grun, N., Scheid, W.: K-shell ionisation in relativistic heavy-ion collisions. J. Phys. B At. Mol. Phys. 20, 2075 (1987) 
36. De Soto, W., Klein, S.A., Beckman, W.A.: Improvement and validation of a model for photovoltaic array performance. Solar Energy 80(1), 78-88 (2006)

37. Sivasankar, G., Kumar, S.V.: Improving low voltage ride through of wind generators using STATCOM under symmetric and asymmetric fault conditions. Int. Rev. Modell. Simul. 6(4), 1212-1218 (2013)

38. Tame, M.S., McEnery, K.R., Özdemir, Ş.K., Lee, J., Maier, S.A., Kim, M.S.: Quantum plasmonics. Nat. Phys. 9, 329-340 (2013)

39. Kamal, E., Koutb, M., Sobaih, A.A., Abozalam, B.: An intelligent maximum power extraction algorithm for hybrid wind-dieselstorage system. Int. J. Electr. Power Energy Syst. 32(3), 170-177 (2010)
40. Pregnolato, T., Lee, E.H., Song, J.D., Stobbe, S., Lodahl, P.: Single-photon non-linear optics with a quantum dot in a waveguide. Nat. Commun. 6, 8655-8659 (2015)

41. Yan, W.-B., Fan, H.: Single-photon quantum router with multiple output ports. Sci. Rep. 4, 4820-4826 (2014)

Publisher's Note Springer Nature remains neutral with regard to urisdictional claims in published maps and institutional affiliations. 